\title{
THE
}

1975

\section{The Interaction of Rare Gas Atoms with Graphite Surfaces. I. Single Adatom Energies}

David L. Freeman

University of Rhode Island, dfreeman@uri.edu

Follow this and additional works at: https://digitalcommons.uri.edu/chm_facpubs

Terms of Use

All rights reserved under copyright.

\section{Citation/Publisher Attribution}

Freeman, D. L. (1975). The Interaction of Rare Gas Atoms with Graphite Surfaces. I. Single Adatom Energies, J. Chem. Phys., 62(3), 941-949. doi: 10.1063/1.430547

Available at: https://doi.org/10.1063/1.430547

This Article is brought to you for free and open access by the Chemistry at DigitalCommons@URI. It has been accepted for inclusion in Chemistry Faculty Publications by an authorized administrator of DigitalCommons@URI. For more information, please contact digitalcommons-group@uri.edu. 
AIP Chemicital Prysics

The interaction of rare gas atoms with graphite surfaces. I. Single adatom energies

David L. Freeman

Citation: J. Chem. Phys. 62, 941 (1975); doi: 10.1063/1.430547

View online: http://dx.doi.org/10.1063/1.430547

View Table of Contents: http://jcp.aip.org/resource/1/JCPSA6/v62/i3

Published by the American Institute of Physics.

Additional information on J. Chem. Phys.

Journal Homepage: http://jcp.aip.org/

Journal Information: http://jcp.aip.org/about/about_the_journal

Top downloads: http://jcp.aip.org/features/most_downloaded

Information for Authors: http://jcp.aip.org/authors

\section{ADVERTISEMENT}

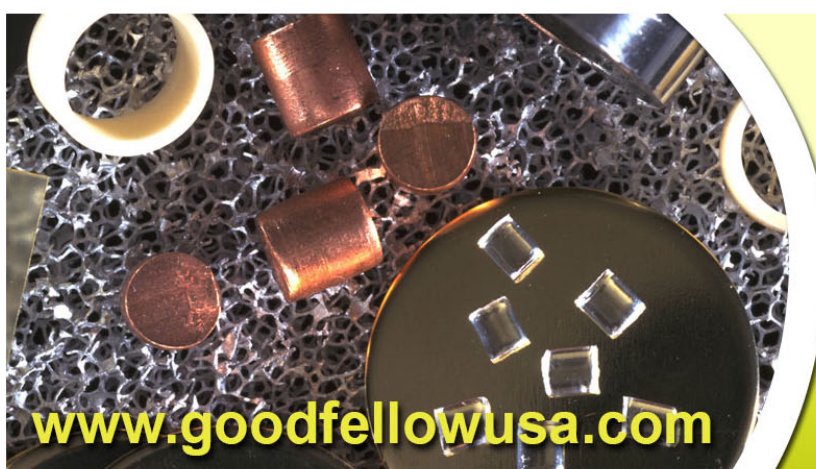




\title{
The interaction of rare gas atoms with graphite surfaces. I. Single adatom energies
}

\author{
David L. Freeman* \\ Battelle Memorial Institute, Columbus, Ohio 43201 \\ (Received 9 August 1974) \\ The Gordon-Kim local density method is applied to the calculation of the interaction energy of \\ helium, neon, argon, and krypton with the basal plane of graphite. In all cases, the binding site is \\ found to be above the center of a hexagon, but the barrier to migration to other sites is less than \\ $50 \mathrm{cal} /$ mole. Comparisons are made with other studies on these systems, and the role of \\ non-two-body additive effects is discussed.
}

\section{INTRODUCTION}

Essential to the understanding of the kinetic and thermodynamic properties of atoms physisorbed on solid surfaces is the determination of the interaction potential between the adatoms and the substrate. Accurate quantum mechanical calculations of the interaction potential in physisorption are very difficult because the system has low symmetry, the number of electrons is large, and the forces between the adatoms and the substrate are quite weak. As a consequence, most studies which have attempted to calculate adatom-surface interactions have employed simplifying assumptions in order to make the problem tractable. ${ }^{1}$

For physisorption studies on insulators, the most commonly used approach is to assume that the net potential is the sum of two-body potentials between the adatom and the surface atoms of the solid. ${ }^{1-14}$ This technique has been applied extensively to the calculation of adsorption potentials between rare gas atoms and the surfaces of rare gas crystals, ${ }^{1-6}$ and to the physisorption of various nonpolar atoms and molecules on graphite, 1, 7-14 For the case of adsorption of rare gas atoms on rare gas crystals, the two-body potential parameters are chosen from gas phase data. The basic errors in such a calculation a rise from the neglect of non-two-body additive interactions. A recent calculation by Bennett of argon adsorbed on argon crystal has shown that the non-two-body additive contributions can alter the binding energy by up to $12 \%$ and modify the barrier to surface migration by $70 \% .{ }^{15}$ Since the barrier to surface migration has an important influence on the thermodynamic properties of thin films, errors as large as $70 \%$, even for small migration barriers, can be quite significant. For the case of rare gas adsorption on graphite, in addition to the errors due to non-two-body additive effects, the choice of potential parameters is ambiguous. As Sams has pointed out, there are a variety of reasonable rare gas-carbon potential parameters that can be used, and the different choices can yield very different results. ${ }^{13}$ For example, when potential parameters were chosen from the combining rules of Fender and Halsey, ${ }^{16}$ the calculated binding energies differed from experiment by as much as $67 \%$, whereas when the combining rules of Hudson and McCoubrey ${ }^{17}$ were used, the binding energies never differed from experiment by more than $10 \% .{ }^{13}$ Since there is no a priori way of choosing one combining rule over another, Sams concluded that the agreement with experiment obtained by many authors using this technique has been fortuitous.

Because graphite is believed to have one of the smoothest surfaces found in nature (i.e., the barrier to surface migration by an adatom is small), there has been considerable experimental and theoretical attention paid to rare gas films on graphite. ${ }^{18-20}$ As a consequence, it is important to determine accurate potential energy surfaces for this system. Because the previous calculations have neglected non-two-body additive effects and have used uncertain parameters, it would be most useful to apply a technique that is free of these problems.

To circumvent many of the difficulties that arise in problems involving the electronic structure of the surface of a solid, a variety of calculations have employed density functional techniques based on the work of Hohenberg and Kohn. ${ }^{21,22}$ Recently, Smith, Ying, and Kohn have applied density functional methods to hydrogen chemisorption on metals, ${ }^{23}$ and Kleiman and Landman have used the method to compute the repulsive interaction between helium atoms and metal surfaces. ${ }^{24,25}$ One of the more successful density functional techniques is due to Gordon and Kim (GK). ${ }^{26}$ The GK method was developed to study intermolecular forces between closedshell species, and it has been successfully applied to interactions between closed-shell atoms and ions. For the rare gas dimers $\mathrm{Ne}_{2}, \mathrm{Ar}_{2}, \mathrm{Kr}_{2}$, and $\mathrm{Xe}_{2}$, the GK method predicts binding energies good to about $10 \%$, and to even better accuracy in the repulsive region of the potential curves. The GK method has also been used to calculate interactions between the rare gases and some simple diatomic molecules. ${ }^{27,28}$ Recently, Bennett applied the GK method to compute the interaction potential between an argon atom and the (100) face of argon crystal. ${ }^{15}$ Since graphite can be regarded as a closed-shell system, it seems natural to apply the GK method to rare gas adsorption on it.

In what follows, we present the results of a GK calculation of the interaction energy between the basal plane of $\mathrm{g}$ raphite and adatoms of helium, neon, argon, and krypton. Because the GK technique fails to give the correct dispersion forces, we do not expect to attain the same accuracy for adsorption of an infinite crystal as GK obtained for atom-atom interactions. However, the GK technique does not require any uncertain parameters, and it does include non-two-body additive contributions. 
The application of the GK method to rare gas adsorption on graphite can be expected to provide more reliable potential surfaces than are presently available.

In Sec. II we briefly review the GK method and discuss our extension of it to the interaction of adatoms with graphite. In Sec. III we give our results, and we present our conclusions in Sec. IV.

\section{APPLICATION OF THE GORDON-KIM METHOD TO RARE GAS-GRAPHITE INTERACTIONS}

The details and assumptions of the GK method are given in Ref. 26, and an analysis of the method has been given by Kim and Gordon. ${ }^{29}$ Here we only discuss the GK method in direct reference to our calculations on graphite surfaces.

The GK method is used to calculate the interaction potential between closed-shell systems in the region of significant overlap of the electronic charge distributions. It is assumed that the charge density of the interacting system is given by the sum of the unperturbed charge densities of its composite closed-shell parts. In this approximation, the charge density for the system of a rare gas atom interacting with a graphite surface is given by

$$
\rho(\mathbf{r})=\rho_{a}(\mathbf{r})+\rho_{s}(\mathbf{r}),
$$

where $\rho(\mathbf{r})$ is the total charge density (including both the electrons and the nuclei), $\rho_{a}(\boldsymbol{r})$ is the atomic charge density, and $\rho_{s}(\boldsymbol{r})$ is the surface charge density of graphite. In their calculations on the interactions between closed-shell atoms, GK took Hartree-Fock densities for the electronic contribution to $\rho_{a}(\mathbf{r})$, and we have done the same. For the electron density of neon and argon, we used the analytic Hartree-Fock wavefunctions of Huzinaga, McWilliams, and Domsky. ${ }^{30}$ The neon basis consisted of $5 s$ orbitals and $4 p$ orbitals, and the argon basis consisted of $8 s$ orbitals and $8 p$ orbitals. The helium and krypton Hartree-Fock wavefunctions were taken from Clementi. ${ }^{31}$ The helium basis consisted of $5 s$ orbitals and the krypton basis consisted of $10 \mathrm{~s}$ orbitals, $9 p$ orbitals, and $5 d$ orbitals. Unfortunately, densities of the same accuracy are not available for graphite. Instead, we generated the electronic contribution to $\rho_{s}(\mathbf{r})$ from the reasonably accurate two-dimensional band wavefunctions of Painter and Ellis. ${ }^{32} \mathrm{~A}$ discussion of the sensitivity of the GK method to the electron density function will be given later in this paper.

By using two-dimensional band wavefunctions, we have modeled our surface by a single plane of carbon atoms. Since the spacing between sheets in graphite is large $(3.37 \AA)$, the energy, due to the other layers, will only affect contributions to the long-range dispersion energy. These contributions are inherently assumed to be zero by the GK technique. The contribution to the GK energy from other than the surface layer of carbon atoms is expected to be negligible, and this single layer approximation therefore should introduce no significant additional errors not already due to the approximations of the GK method.

From Ref. 32, the wavefunction for the $m$ th band state at crystal momentum $\mathbf{k}$ and point $\mathbf{r}$ is given by

$$
\psi_{m}(\mathbf{k}, \mathbf{r})=\sum_{j, \mu} A_{m j}(\mathbf{k}) e^{i \mathbf{k} \cdot \mathbf{R}_{\mu}} \varphi_{j}\left(\mathbf{r}-\mathbf{R}_{\mu}-\alpha_{j}\right),
$$

where $\mu$ sums over the unit cells located at $\mathbf{R}_{\mu}$ in the two-dimensional lattice, $j$ sums over the Slater basis functions $\varphi_{j}\left(\mathbf{r}-\mathbf{R}_{\mu}-\alpha_{j}\right)$ centered at $\mathbf{R}_{\mu}+\alpha_{j}$, $\alpha$ locates each of the two carbon atoms in a graphite unit cell, and the $A_{m j}(\mathbf{k})$ are expansion coefficients. Details concerning the basis set used, the geometry of the graphite lattice, and the number of terms included in the sum on $\mu$ in Eq. (2) can be found in Ref. 32. Defining $\bar{\rho}_{s}(\mathbf{r})$, to be the electronic contribution to $\rho_{s}(\mathbf{r})$, we have

$$
\bar{\rho}_{s}(\mathbf{r})=\sum_{m} \int d^{2} k\left|\psi_{m}(\mathbf{k}, \mathbf{r})\right|^{2}
$$

Using Eqs. (2) and (3), we can write

$$
\bar{\rho}_{s}(\mathbf{r})=\sum_{\substack{i, j \\ \mu, \nu}} \Gamma_{i j}^{\mu \nu} \varphi_{i}\left(\mathbf{r}-\mathbf{R}_{\nu}-\alpha_{i}\right) \varphi_{j}\left(\mathbf{r}-\mathbf{R}_{\mu}-\alpha_{j}\right),
$$

where the density matrix $\Gamma_{i j}^{\mu \nu}$ is given by

$$
\Gamma_{i j}^{\mu \nu}=\sum_{m} \int d^{2} k A_{m i}^{*}(\mathbf{k}) A_{m j}(\mathbf{k}) \exp [i k \cdot(\mathbf{R} \mu-\mathbf{R} \nu)] .
$$

We found that the direct evaluation of $\mathrm{Eq}$. (4) was too time-consuming for practical GK calculations. To make the GK calculation possible, we found it necessary to fit the graphite density obtained from Eq. (4) to a function that could be easily evaluated. We divided one of the 12 equivalent sectors of a graphite unit cell, shown in Fig. 1, into 1026 regions and fit 254 evaluated grid points [from Eq. (4)] in each region to a function of the form

$$
F(x, y, z)=\sum_{i=1}^{3} \sum_{j, k, l=0}^{2} B_{i j k l} e^{-\beta_{i} R} x^{j} y^{k} z^{l} .
$$

In Eq. (6), $R$ is the distance between the carbon atom located at position $B$ in Fig. 1 and the point at $(x, y, z)$ in the shaded region of Fig. 1. The nonlinear parameters $\beta_{i}$ were optimized, and the coefficients $B_{i j k l}$ were chosen by a least squares fit to the grid points of the function $F$. It was found that $F(x, y, z)$ approximated $\bar{\rho}_{s}(\mathbf{r})$ to three significant figures. Since the expansion coefficients $A_{m j}(\mathbf{k})$ supplied by Painter and Ellis were also given to three figures, we considered this fit adequate.

In the GK method, the interaction energy is computed

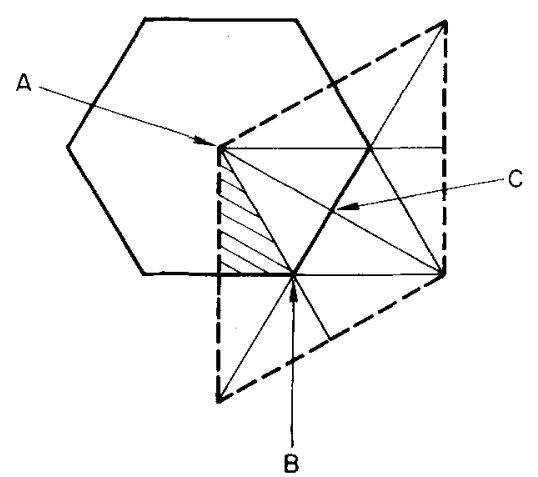

FIG. 1. One hexagon of the two-dimensional graphite lattice is shown. The dashed line is a graphite unit cell, and the cell is divided into its 12 equivalent sectors. The graphite density function was fit in the shaded region. 
from a local functional of the density given in Eq. (1). The Coulombic contribution to the interaction energy is given by the classical expression

$$
V_{c}\left(\mathbf{R}_{\mathbf{u}}\right)=\int \frac{\rho_{a}\left(\mathbf{r}^{\prime}\right) \rho_{s}}{\left|\mathbf{r}-\mathbf{r}^{\prime}\right|} \frac{(\mathbf{r})}{\prime} d^{3} r d^{3} r^{\prime}
$$

which we write

$$
V_{c}\left(\mathbf{R}_{a}\right)=\int P(\mathbf{r}) \rho_{s}(\mathbf{r}) d^{3} r
$$

where

$$
P(\mathbf{r})=\int \frac{\rho_{a}\left(\mathbf{r}^{\prime}\right)}{\left|\mathbf{r}-\mathbf{r}^{\prime}\right|} d^{3} r^{\prime}
$$

$\mathbf{R}_{a}$ is the position of the adatom relative to the surface. Since $\rho_{s}(\mathbf{r})$ is periodic in $\mathbf{r}$, we have

$$
V_{c}\left(\mathbf{R}_{a}\right)=\sum_{\mu} \int_{c} P\left(\mathbf{r}+\mathbf{R}_{\mu}\right) \rho_{s}(\mathbf{r}) d^{3} r,
$$

where the $c$ in Eq. (10) implies integration over one unit cell. From Eq. (10) and

$$
\rho_{s}(\mathbf{r})=6 \sum_{\mu, i} \delta\left(\mathbf{r}-\mathbf{R}_{\mu}-\alpha_{i}\right)-\bar{\rho}_{s}(\mathbf{r})
$$

we have

$$
V_{c}\left(\mathbf{R}_{a}\right)=-\sum_{\mu} \int_{c} d^{3} r \bar{\rho}_{s}(\mathbf{r})\left[P\left(\mathbf{r}+\mathbf{R}_{\mu}\right)-\frac{1}{2} P\left(\alpha_{1}\right)-\frac{1}{2} P\left(\alpha_{2}\right)\right] .
$$

The kinetic, exchange, and correlation contributions to the interaction energy are given by

$$
\begin{aligned}
E_{\boldsymbol{g}}\left(\mathbf{R}_{a}\right)= & \int d^{3} r\left\{\bar{\rho}(\mathbf{r}) E_{G}(\bar{\rho}(\mathbf{r}))\right. \\
& \left.-\bar{\rho}_{a}(\mathbf{r}) E_{G}\left(\bar{\rho}_{a}(\mathbf{r})\right)-\bar{\rho}_{s}(\mathbf{r}) E_{G}\left(\bar{\rho}_{s}(\mathbf{r})\right)\right\},
\end{aligned}
$$

where $\bar{\rho}(\mathbf{r})$ and $\bar{\rho}_{a}(\mathbf{r})$ are, respectively, the electronic contributions to the total and atomic charge densities, and

$$
E_{G}(\rho)=\frac{3}{10}\left(3 \pi^{2}\right)^{2 / 3} \rho^{2 / 3}-\frac{3}{4}(3 / \pi)^{1 / 3} \rho^{1 / 3}+E_{c}(\rho) .
$$

The first term in Eq. (14) is the kinetic energy contribution, and the second term is the exchange energy. The correlation energy $E_{c}(\rho)$ is given by

$$
\begin{aligned}
& E_{c}(\rho)=-0.438 r_{s}^{-1}+1.325 r_{s}^{-3 / 2}-1.47 r_{s}^{-2}-0.4 r_{s}^{-5 / 2}, r_{s}>10 ; \\
& E_{c}(\rho)=0.01898 \ln r_{s}-0.06156,10>r_{s}>0.7 ; \\
& E_{c}(\rho)=0.0311 \ln r_{s}-0.048+0.009 r_{s} \ln r_{s}-0.01 r_{s}, 0.7>r_{s},
\end{aligned}
$$

where

$$
r_{s}=(3 / 4 \pi \rho)^{1 / 3} \text {. }
$$

Combining Eqs. (12) and (13), the total interaction energy is given by

$$
E\left(\mathbf{R}_{a}\right)=\sum_{\mu} I_{\mu}\left(\mathbf{R}_{a}\right)
$$

where

$$
I_{\mu}\left(\mathbf{R}_{a}\right)=\int_{c} d^{3} r\left\{\bar{\rho}\left(\mathbf{r}+\mathbf{R}_{\mu}\right) E_{G}\left(\bar{\rho}\left(\mathbf{r}+\mathbf{R}_{\mu}\right)\right)-\bar{\rho}_{a}\left(\mathbf{r}+\mathbf{R}_{\mu}\right) E_{G}\left(\bar{\rho}_{a}\left(\mathbf{r}+\mathbf{R}_{\mu}\right)\right)-\bar{\rho}_{s}(\mathbf{r})\left[P\left(\mathbf{r}+\mathbf{R}_{\mu}\right)+E_{G}\left(\bar{\rho}_{s}(\mathbf{r})\right)-\frac{1}{2} P\left(\boldsymbol{\alpha}_{1}\right)-\frac{1}{2} P\left(\boldsymbol{\alpha}_{2}\right)\right]\right\}
$$

In Eq. (18) we have combined all contributions to $I_{\mu}\left(\mathbf{R}_{a}\right)$ into a single three-dimensional integral. The natural coordinate system for performing the integration in Eq. (18) is Cartesian coordinates, and the coordinate axes are defined in Figs. $2(a)$ and $2(b)$. In Fig. 2, the twodimensional graphite lattice defines the $(x, y)$ plane, a possible location for the adatom is denoted by an asterisk, and unit cell $\mu$ is shown in Fig. 2(a). The integrations over the $x$ and $y$ coordinates were performed using Gauss-Legendre quadrature. For the $z$ integration, we used Gauss-Legendre quadrature between $z=0$ and the location of the adatom. For the region between $z=-\infty$ and $z=0$, and the region between the adatom and $z=+\infty$, we used Gauss-Laguerre quadrature. A sufficient number of quadrature points were used to insure convergence to three significant figures for $I_{\mu}$.

Since contributions to the integral of Eq. (18) fall off with the overlap between the closed-shell component parts, and the overlap falls off roughly exponentially, the series in Eq. (17) converges rapidly. In our calculations we retained enough terms in Eq. (17) so that $E\left(\mathrm{R}_{a}\right)$ would be accurate to three significant figures. We found that it was sufficient in all cases studied to sum over all unit cells within $8 \mathrm{a} . \mathrm{u}$. of the projected position of the adatom on the $(x, y)$ plane.

\section{RESULTS}

To explore the potential surface for rare gas-graphite interactions, we computed the energy as a function of $z$ from Eq. (17) at three positions above the graphite lattice. The positions, shown in Fig. 1, are over the center of the hexagon (position $A$ ), above a carbon atom (position $B$ ), and over the center of a carbon-carbon bond (position $C$ ). In Tables $I-I V$, we have tabulated the interaction energy as a function of $z$ for the three positions for helium, neon, argon, and krypton, and we have plotted these results in Figs. 3-6. As expected, the most strongly bound position for each rare gas atom is at the center of the hexagon, but the barrier to migration to different sites over the graphite lattice is quite small. To understand the significance of the calculation, we have summarized some of the properties of these systems in Table $\mathrm{V}$. In Table $\mathrm{V}$, we have defined $Z_{0 A}$ $\left(Z_{0 B}\right.$ or $\left.Z_{0 C}\right)$ to be the vertical position of minimum energy for position $A(B$ or $C)$, and $Z_{A}^{\prime}\left(Z_{B}^{\prime}\right.$ or $\left.Z_{C}^{\prime}\right)$ to be the value of $z$ over position $A$ ( $B$ or $C$ ) for which the interaction potential crosses zero energy. The values of $Z_{0}$ were determined to within 0.1 a.u., and the values of $Z^{\prime}$ were determined to within 0.2 a. u. If we let $\bar{E}_{A}\left(\bar{E}_{B}\right.$ or $\left.\bar{E}_{C}\right)$ be $E\left(Z_{0 A}\right)\left[\left(E\left(Z_{0 B}\right)\right.\right.$ or $\left.E\left(Z_{0 C}\right)\right]$ for neon, then we have defined the relative well depth $S_{I}$ by

$$
S_{I}=E\left(Z_{0 I}\right) / \bar{E}_{I}
$$

for $I=A, B$, or $C$. We have also defined the barriers to migration $\Delta E_{A B}, \Delta E_{A C}$, and $\Delta E_{B C}$ by

$$
\Delta E_{I J}=E\left(Z_{0 I}\right)-E\left(Z_{0 J}\right)
$$

for $I, J=A, B$, or $C$. The numbers in parentheses in Table $\mathrm{V}$ are taken from the calculations of Steele, ${ }^{14}$ and of 

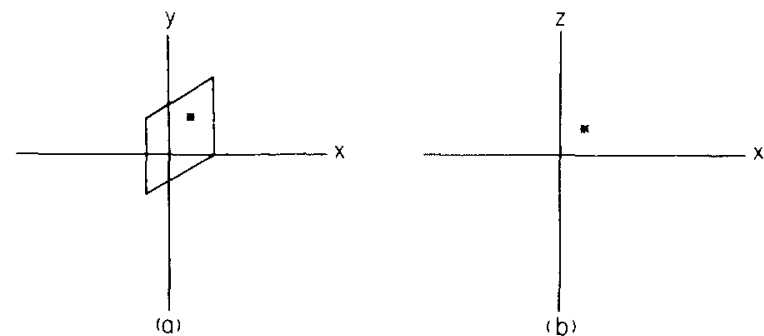

FIG. 2. The coordinates used for the integrations are defined with the graphite lattice in the $(x, y)$ plane. The asterisk denotes a possible position for the adatom.

Crowell and Steele, ${ }^{11}$ where the net potential is taken to be the sum of two-body 6-12 Lennard-Jones potentials. We see that there is fair agreement between the results of the GK method and the two-body additive method for the binding positions and relative well depths. The migration barriers differ by up to $50 \%$, and as Bennett has shown, this sort of difference can be expected from nontwo-body additive effects. ${ }^{15}$ The well depths determined in the present calculation are substantially lower than those found by the other method. To see this more clearly, in Fig. 7, we have plotted the interaction energy of argon on graphite over position $A$ of Fig. 1 using the two-body additive method and the results of the present calculation. Curve $A$ of Fig. 7 is the GK result, and curve $B$ was computed from Eq. (2.33) of Ref. 14. The parameters used in computing curve $B$ of $\mathrm{Fig} .7$ were taken from Steele, ${ }^{33}$ and the se parameters are known to yield binding energies that are in reasonable agreement with experiment (perhaps fortuitously; see Ref. 13). The poor agreement between our work and Steele's work with respect to the well depths makes it necessary to examine the errors in the present calculation in some detail. In the following discussion of our errors, we assume that the well depths given in Ref. 14 are correct, and we show that errors due to dispersion

TABLE I. Helium on graphite. ${ }^{2}$

\begin{tabular}{rlll}
\hline \hline$Z^{\mathrm{b}}$ & $E\left(Z_{A}\right)^{\mathrm{b}}$ & $E\left(Z_{B}\right)^{\mathrm{b}}$ & $E\left(Z_{C}\right)^{\mathrm{b}}$ \\
\hline 3.0 & 0.0254 & 0.0510 & 0.0475 \\
4.0 & 0.00344 & 0.00682 & 0.00646 \\
4.4 & 0.00100 & & \\
4.6 & 0.000370 & 0.00126 & 0.00117 \\
4.8 & -0.0000206 & 0.000534 & 0.000482 \\
5.0 & -0.000243 & 0.0000974 & 0.0000665 \\
5.2 & -0.000355 & -0.000149 & -0.000167 \\
5.3 & -0.000381 & -0.000227 & -0.000236 \\
5.4 & -0.000394 & -0.000272 & -0.000282 \\
5.5 & -0.000395 & -0.000303 & -0.000311 \\
5.6 & -0.000389 & -0.000319 & -0.000325 \\
5.7 & -0.000377 & -0.000325 & -0.000329 \\
5.8 & -0.000360 & -0.000321 & -0.000324 \\
6.0 & -0.000318 & -0.000298 & -0.000299 \\
7.0 & -0.000119 & -0.000121 & -0.000120 \\
8.0 & -0.0000327 & -0.0000334 & -0.0000334 \\
9.0 & -0.00000807 & -0.00000821 & -0.00000819 \\
10.0 & -0.00000187 & -0.00000187 & -0.00000187 \\
\hline \hline
\end{tabular}

aAll values expressed in atomic units.

${ }^{\mathrm{b}} Z_{A}\left(Z_{B}\right.$ or $\left.Z_{C}\right)$ is the vertical adatom distance over position $A$ $(B$ or $C)$ of Fig. 1 .
TABLE II. Neon on graphite. ${ }^{2}$

\begin{tabular}{cccc}
\hline \hline$Z^{\mathrm{b}}$ & $E\left(Z_{A}\right)^{\mathrm{b}}$ & $E\left(Z_{B}\right)^{\mathrm{b}}$ & $E\left(Z_{C}\right)^{\mathrm{b}}$ \\
\hline 3.0 & 0.0892 & 0.170 & 0.158 \\
4.0 & 0.0154 & 0.0262 & 0.0250 \\
5.0 & 0.00111 & 0.00231 & 0.00219 \\
5.2 & 0.000422 & 0.00116 & 0.00109 \\
5.4 & 0.00000876 & 0.000468 & 0.000424 \\
5.5 & -0.000124 & & 0.000201 \\
5.6 & -0.000220 & 0.0000593 & 0.0000331 \\
5.8 & -0.000330 & -0.000162 & -0.000179 \\
5.9 & -0.000355 & -0.000226 & -0.000237 \\
6.0 & -0.000366 & -0.000267 & -0.000275 \\
6.1 & -0.000366 & -0.000290 & -0.000297 \\
6.2 & -0.000358 & -0.000301 & -0.000306 \\
6.3 & -0.000345 & -0.000302 & -0.000306 \\
6.4 & -0.000328 & -0.000296 & -0.000299 \\
6.5 & -0.000308 & -0.000285 & -0.000286 \\
6.6 & -0.000287 & -0.000270 & -0.000271 \\
7.0 & -0.000200 & -0.000196 & -0.000196 \\
8.0 & -0.0000599 & -0.0000610 & -0.0000608 \\
9.0 & -0.0000152 & -0.0000154 & -0.0000154 \\
10.0 & -0.00000350 & -0.00000353 & -0.00000353 \\
\hline \hline
\end{tabular}

all values expressed in atomic units.

${ }^{b} Z_{A}\left(Z_{B}\right.$ or $\left.Z_{C}\right)$ is the vertical adatom distance over position $A(B$ or $C)$ of $\mathrm{Fig} .1$

contributions and basis set deficiencies in our results are of the right magnitude to explain the discrepancies of Fig. 7.

As we indicated, the GK method has proved to be reliable to about $10 \%$ for closed-shell atom-atom interactions in the region of the potential minimum and where the potential is repulsive. Since $E(R)$ becomes zero as the overlap between the two closed-shell electronic sys-

TABLE III. Argon on graphite. ${ }^{a}$

\begin{tabular}{llcc}
\hline \hline$Z^{\mathrm{b}}$ & $E\left(Z_{A}\right)^{\mathrm{b}}$ & $E\left(Z_{B}\right)^{\mathrm{b}}$ & $E\left(Z_{C}\right)^{\mathrm{b}}$ \\
\hline 3.0 & 0.208 & 0.297 & 0.279 \\
4.0 & 0.0490 & 0.0641 & 0.0619 \\
5.0 & 0.00689 & 0.00927 & 0.00897 \\
5.5 & 0.00142 & 0.00229 & 0.00219 \\
5.75 & 0.000200 & & \\
5.8 & 0.0000328 & 0.000503 & 0.000438 \\
5.85 & -0.000114 & & \\
5.9 & -0.000243 & & \\
5.95 & -0.000355 & & -0.000183 \\
6.0 & -0.000451 & -0.000145 & -0.000540 \\
6.2 & -0.000713 & -0.000515 & -0.000714 \\
6.4 & -0.000826 & -0.000700 & -0.000756 \\
6.5 & -0.000844 & -0.000745 & \\
6.59 & -0.000845 & & -0.000774 \\
6.6 & -0.000845 & -0.000765 & \\
6.64 & -0.000840 & & -0.000774 \\
6.7 & -0.000829 & -0.000766 & -0.000759 \\
6.8 & -0.000803 & -0.000754 & -0.000705 \\
7.0 & -0.000732 & -0.000701 & -0.000320 \\
8.0 & -0.000322 & -0.000320 & -0.000105 \\
9.0 & -0.000105 & -0.000105 & -0.0000299 \\
10.0 & -0.0000299 & -000299 \\
\hline \hline
\end{tabular}

${ }^{2}$ All values expressed in atomic units.

${ }^{\mathrm{b}} Z_{A}\left(Z_{B}\right.$ or $\left.Z_{C}\right)$ is the vertical adatom distance over position $A(B$ or $C)$ of Fig. 1 . 
TABLE IV. Krypton on graphite. ${ }^{a}$

\begin{tabular}{cccc}
\hline \hline$Z^{\mathrm{b}}$ & $E\left(Z_{A}\right)^{\mathrm{b}}$ & $E\left(Z_{B}\right)^{\mathrm{b}}$ & $E\left(Z_{C}\right)^{\mathrm{c}}$ \\
\hline 3.0 & 0.289 & 0.415 & 0.390 \\
4.0 & 0.0741 & 0.0928 & 0.0903 \\
5.0 & 0.0126 & 0.0156 & 0.0153 \\
6.0 & 0.000113 & 0.000541 & 0.000492 \\
6.1 & -0.000227 & 0.000122 & 0.0000822 \\
6.15 & & -0.0000514 & -0.0000877 \\
6.2 & -0.000488 & -0.000204 & -0.000237 \\
6.3 & -0.000685 & & \\
6.4 & -0.000827 & -0.000640 & -0.000662 \\
6.5 & -0.000925 & & -0.000878 \\
6.6 & -0.000986 & -0.000865 & -0.000931 \\
6.7 & -0.00102 & -0.000920 & -0.00957 \\
6.8 & -0.00103 & -0.000947 & -0.000961 \\
6.9 & -0.00102 & -0.000954 & -0.000949 \\
7.0 & -0.000994 & -0.000943 & -0.000892 \\
7.1 & -0.000961 & & -0.000807 \\
7.2 & -0.000921 & -0.000888 & -0.000710 \\
7.4 & -0.000825 & -0.000802 & -0.000515 \\
7.6 & -0.000721 & -0.000708 & -0.000187 \\
8.0 & -0.000519 & -0.000514 & -0.0000564 \\
9.0 & -0.000187 & -0.000187 & \\
10.0 & -0.0000564 & -0.0000564 & \\
\hline \hline & & & \\
\hline
\end{tabular}

${ }^{2}$ All values expressed in atomic units.

${ }^{b} Z_{A}\left(Z_{B}\right.$ or $\left.Z_{C}\right)$ is the vertical adatom distance over position $A$ $(B$ or $C)$ of Fig. 1.'

tems goes to zero, it is clear that $E(R)$ is wrong in the dispersive region, where the interaction energy should fall off inversely to the sixth power of the interatomic separation for atom-atom interactions. For adatomsurface interactions, where we only include the dispersive contribution to the attractive term, the energy should fall off as the cube of the adatom surface distance. Although this incorrect treatment of the dispersion energy by the GK method seems to have only a small effect on the binding energy for diatomic systems, the result of adding a large number of terms in the infinite system we have considered might be significant. To estimate the magnitude of the dispersion error in our results,

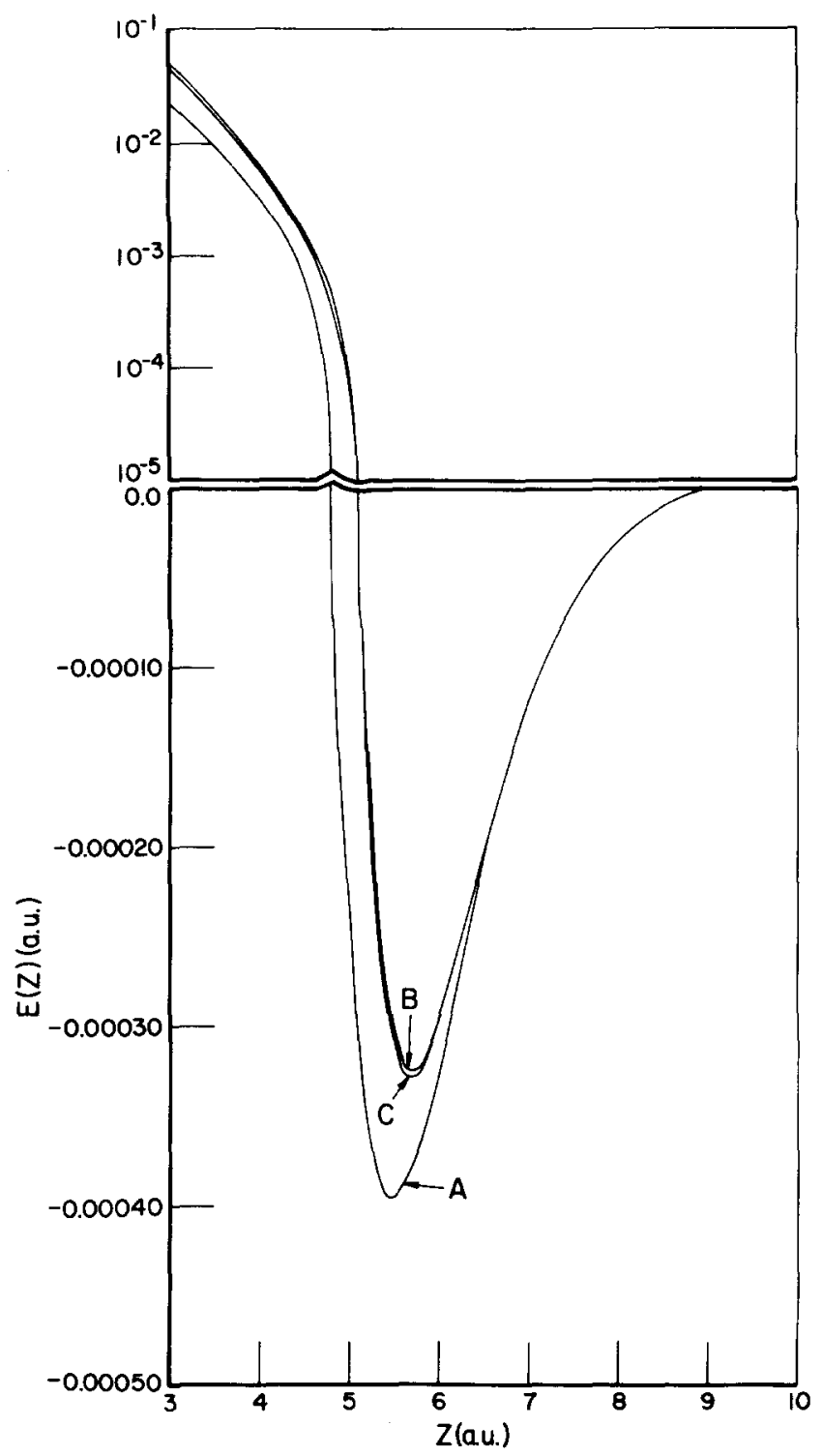

FIG. 3. The interaction energy for helium on graphite as a function of the adatom-surface distance. The positions $A, B$, and $C$ are defined in Fig. 1.

TABLE V. Properties of rare gas-graphite systems. ${ }^{2, b}$

\begin{tabular}{llllr}
\hline \hline & $\mathrm{He}$ & $\mathrm{Ne}$ & $\mathrm{Ar}$ & $\mathrm{Kr}$ \\
\hline$Z_{O A}$ & $5.5(5.5)^{\mathrm{c}}$ & $6.0(5.8)^{\mathrm{c}}$ & $6.6(6.4)^{\mathrm{c}}$ & $6.8(6.6)^{\mathrm{c}}$ \\
$Z_{O B}$ & 5.7 & 6.3 & 6.7 & 6.9 \\
$Z_{O C}$ & 5.7 & 6.3 & 6.7 & 6.9 \\
$Z_{A}^{\prime}$ & 4.7 & 5.4 & 5.8 & 6.1 \\
$Z_{B}^{\prime}$ & 5.1 & 5.7 & 5.9 & 6.1 \\
$Z_{C}^{\prime}$ & 5.1 & 5.7 & 5.9 & 6.1 \\
$E\left(Z_{O A}\right)$ & $-0.000395(-0.000798)$ & $-0.000366(-0.00162)$ & $-0.000845(-0.00361)$ & $-0.00103(-0.00435)$ \\
$E\left(Z_{O B}\right)$ & $-0.000325(-0.000737)$ & $-0.000302(-0.00152)$ & $-0.000766(-0.00349)$ & $-0.000954(-0.00422)$ \\
$E\left(Z_{O C}\right)$ & $-0.000329(-0.000744)$ & $-0.000306(-0.00153)$ & $-0.000774(-0.00351)$ & $-0.000961(-0.00423)$ \\
$S_{A}$ & $1.08(0.492)$ & $1.0(1.0)$ & $2.31(2.23)$ & $2.81(2.68)$ \\
$S_{B}$ & $1.08(0.484)$ & $1.0(1.0)$ & $2.54(2.29)$ & $3.16(2.78)$ \\
$S_{C}$ & $1.08(0.485)$ & $1.0(1.0)$ & $2.53(2.29)$ & $3.14(2.76)$ \\
$\Delta E_{B A}$ & $0.000070(0.000054)$ & $0.000064(0.000088)$ & $0.000079(0.00010)$ & $0.000076(0.00012)$ \\
$\Delta E_{C A}$ & $0.000066(0.000062)$ & $0.000060(0.000099)$ & $0.000071(0.00012)$ & $0.000069(0.00013)$ \\
$\Delta E_{B C}$ & $0.000004(0.000007)$ & $0.000004(0.000011)$ & $0.000008(0.000015)$ & $0.000007(0.000011)$ \\
\hline \hline
\end{tabular}

${ }^{a}$ All values expressed in atomic units.

DThe numbers in parentheses are taken from Ref. 14 unless otherwise indicated.

${ }^{\mathrm{c}}$ Reference 11. 


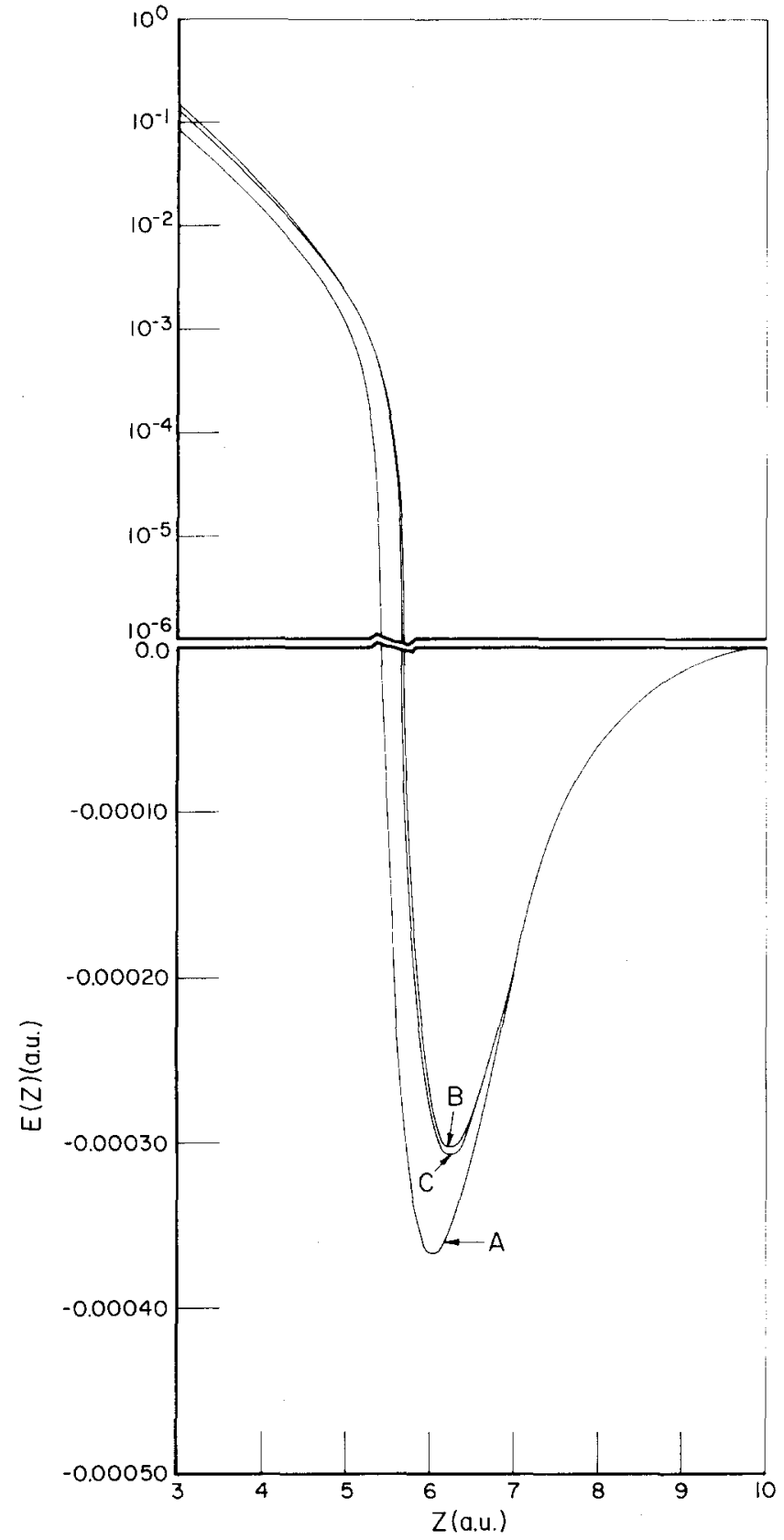

FIG. 4. The interaction energy for neon on graphite as a function of the adatom-surface distance. The positions $A, B$, and $\mathrm{C}$ are defined in Fig. 1.

we followed Steele ${ }^{14}$ and assumed the net raregas-graphite potential to be the sum of two-body 6-12 potentials; i.e., we assumed an interaction energy of the form

$$
\bar{E}\left(\mathbf{R}_{a}\right)=\sum_{j} 4 \mathcal{E}_{g s}\left\{\left(\frac{\sigma_{g s}}{r_{a j}}\right)^{12}-\left(\frac{\sigma_{g s}}{r_{a j}}\right)^{\theta}\right\},
$$

where $r_{a j}$ is the distance between the adatom and the $j$ th carbon atom, and $\mathcal{E}_{g s}$ and $\sigma_{g s}$ are Lennard-Jones parameters which we have taken from Steele. ${ }^{33}$ We considered the case of an argon atom at its equilibrium position (as found by Steele) and summed Eq. (21) over all the carbon atoms that we included in the GK calculation. The difference between this result and the total result given in Ref. 14 gives a measure of the dispersion error in the present calculation due to all carbon atoms in the sur face layer and all other layers that the GK method inherently assumes not to contribute to the interaction energy. For the case of argon at equilibrium, we found an error of $0.00103 \mathrm{a} . \mathrm{u}$. or $29 \%$ of the total. Althouth this dispersion error is substantial, it does not completely explain the apparent error of $76 \%$ which we found in the present calculation relative to Steele's results. ${ }^{14}$

The other major source of error which we consider is the graphite density function. To obtain the excellent results for atom-atom interactions, GK used HartreeFock density functions generated from basis sets at the Hartree-Fock limit. The Painter and Ellis basis set consisted of one function for the core electrons and was of double zeta quality for the valence electrons. ${ }^{32}$ Additionally, the exponents were not optimized. It is expected that a small basis set will yield a density function that is roughly correct near the nucleus, where the contribution to the Hartree-Fock energy is greatest but too small in the asymptotic tail. This incorrect behavior

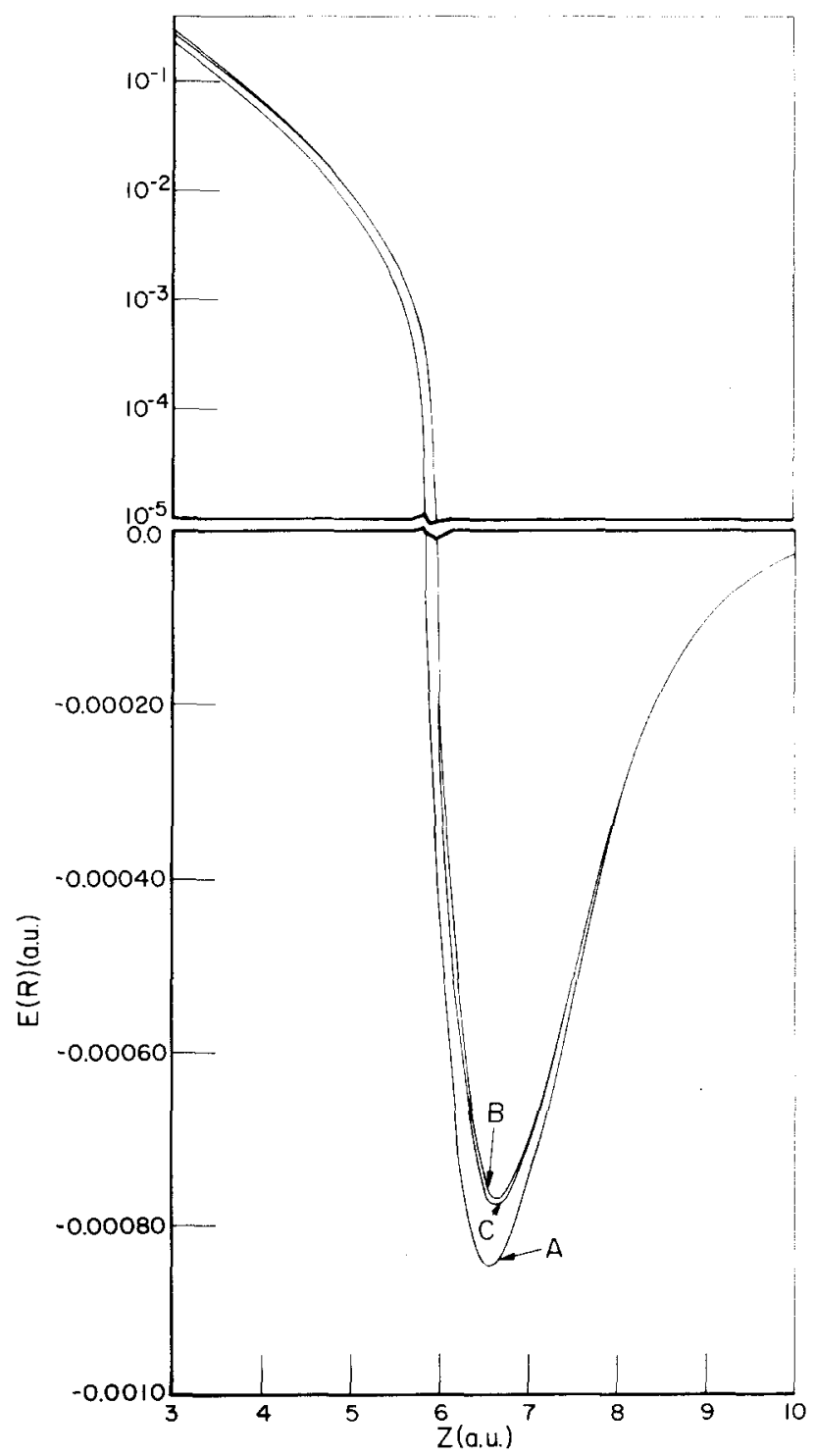

FIG. 5. The interaction energy for argon on graphite as a function of the adatom-surface distance. The positions $A, B$, and $C$ are defined in Fig. 1. 


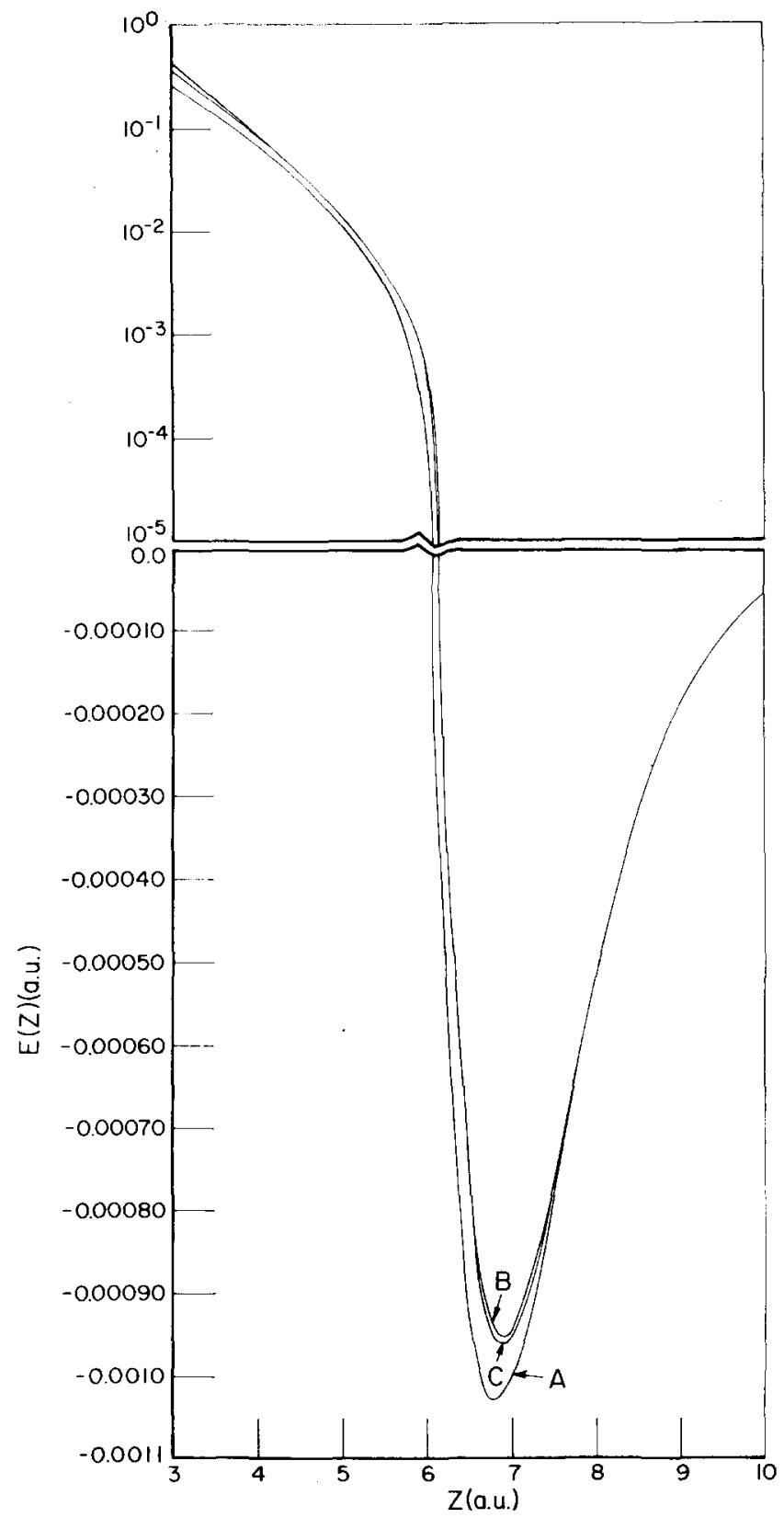

FIG. 6. The interaction energy for krypton on graphite as a function of the adatom-surface distance. The positions $A, B$, and $C$ are defined in Fig. 1.

can be expected to give poor GK results.

To explore the magnitude of the errors we might expect from the use of small basis sets, we performed a GK calculation on $\mathrm{Ar}_{2}$ with a minimum basis consisting of $3 s$ orbitals and $2 p$ orbitals, and the extended basis used in the argon-graphite calculations. Both basis sets were taken from Huzinaga, McWilliams, and Domsky. ${ }^{30}$ The results of this calculation are plotted in Fig. 8, where curve $A$ is the extended basis result and curve $B$ is the result of the minimum basis calculation. We note that the well depth for the minimum basis set calculation is in error by about $68 \%$, and the equilibrium internuclear separation is in error by about $0.5 \mathrm{a} . \mathrm{u}$. The minimum basis also gives too large a repulsive interaction. In the adatom-graphite calculations, the adatom electron density is much more accurate than the graphite electron density. Closer to this situation is the interaction between an argon atom in an extended basis and one in a minimum basis. The results of this calculation are given in curve $C$ of Fig. 8. In this case, the equilibrium geometry is the same as predicted by the extended basis results of curve $A$, but the well depth is still in error by about $56 \%$. This well depth error is quite large, and it is clear that we can blame the difference between the results of this work and that of Ref. 14 on both an inadequate graphite density function and dispersion errors. We expect that the equilibrium geometries computed in this work are reasonably accurate.

If we assume that the well depths found by Steele are correct and that $29 \%$ of the difference between our results and Steele's is due to dispersion, then $47 \%$ of the error is attributable to the density function. This error is smaller than the error we found for the $\mathrm{Ar}_{2}$ interaction potential computed with one argon atom in a minimum basis and one in an extended basis. This fact implies that the graphite density function is of intermediate quality. As we have indicated, we expect that the asymptotic tail of this density will go to zero too quickly, but we expect the density at intermediate distances to

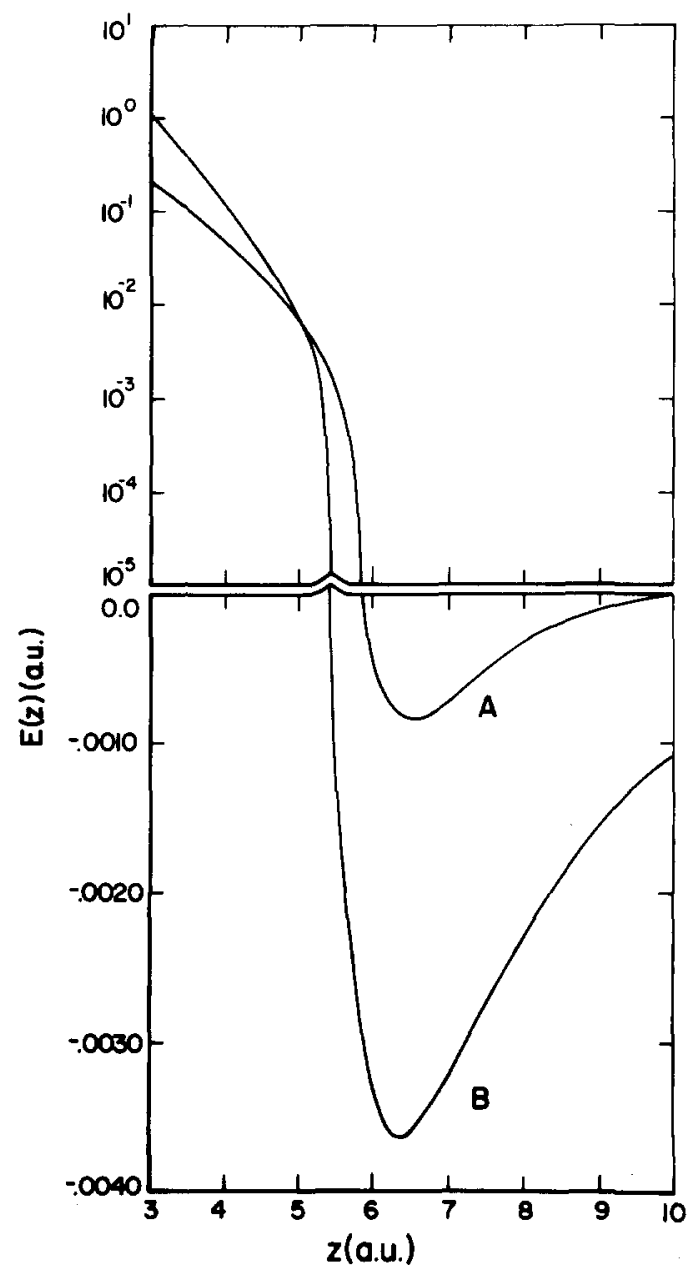

FIG. 7. The interaction energy of an argon atom on graphite over position $A$ of Fig. 1 as a function of adatom-surface distance. Curve $A$ is the result of the present calculation and curve $B$ was computed from Eq. (2.33) of Ref. 14 . 


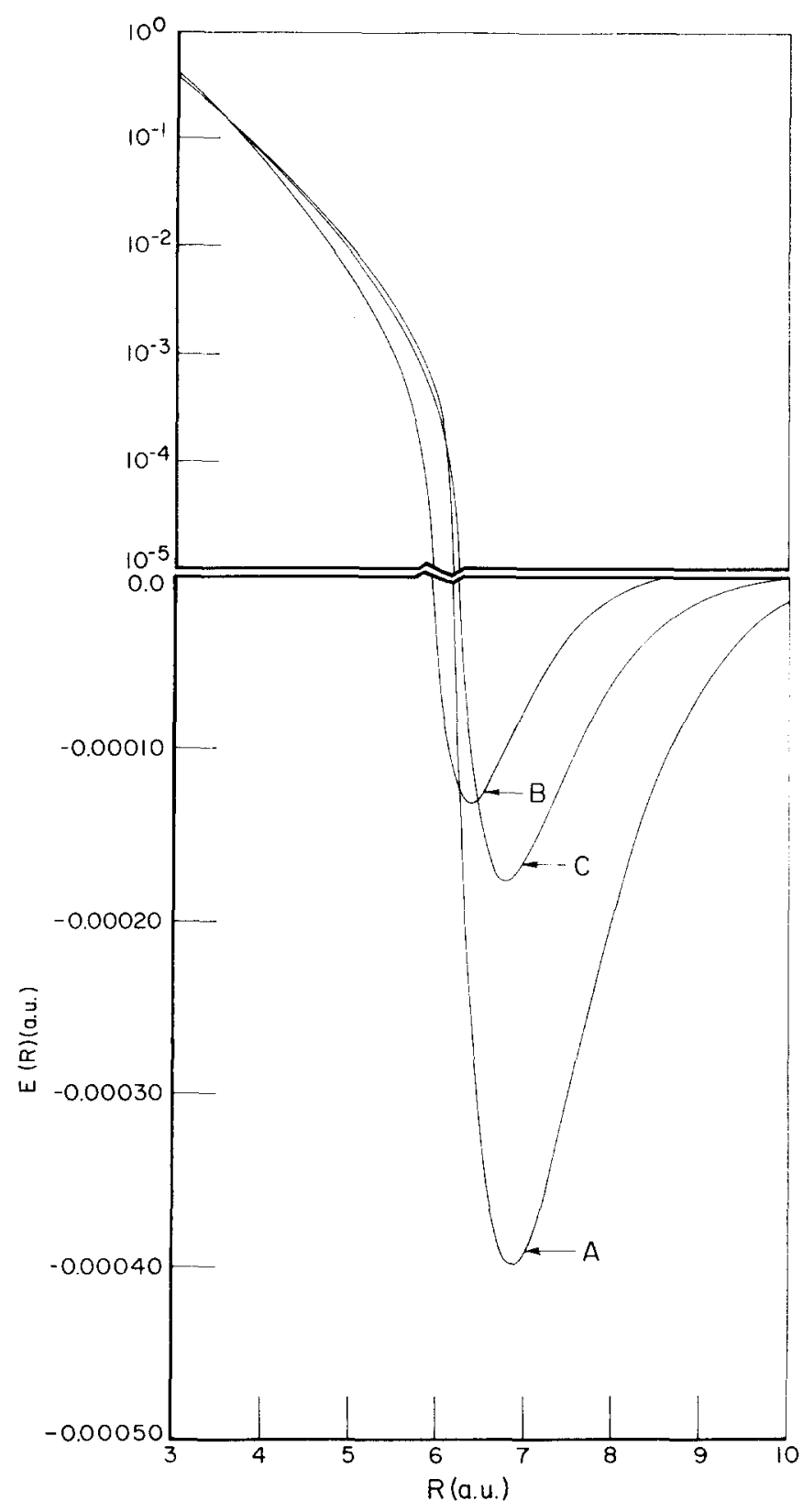

FIG. 8. Curve $A$ is the extended basis argon-argon interaction potential, and curve $B$ is the minimum basis result. Curve $C$ was computed with one argon atom in an extended basis and one in a minimum basis.

be fairly accurate. It is clear that at distances far from the surface, the variation of the density with respect to coordinates parallel to the plane of the surface (i.e., $x$ or $y$ in Fig. 2) will be zero. Most of the contribution to the migration barrier will come from the density nearer to the surface, where it is more accurate. Because the migration barrier should depend on the adatom's local environment, we would expect the dispersion error to be roughly independent of the adatom's $(x, y)$ coordinates. This local dependence of the migration barrier explains why we appear to have more accuracy for it than for the well depths.

With the exception of helium, there is also good agreement between this calculation and that of Ref. 14 for the relative well depths. This is not surprising, since we would expect the relative well depths to depend most strongly on the relative atomic densities, which are quite accurate. The anomalous behavior of helium was also observed for helium-helium interactions. ${ }^{26}$

In summary, two sources of error in the present calculation are due to an inadequate graphite density function and an improper treatment of dispersion forces by the GK method. These errors have yielded well depths that are too small. We expect other properties that depend on the adatom's local environment, such as migration barriers and equilibrium geometries, to be reasonably accurate.

\section{CONCLUSIONS}

The GK method has been successfully applied to the calculation of relative well depths, barriers to migration, and equilibrium geometries for rare gas adsorption on graphite. Because of the incorrect treatment of dispersion forces by the GK method and because of an inadequate graphite density function, the present calculation obtained only about $25 \%$ of the well depths found by other authors. ${ }^{11,14}$ The desirability of repeating this calculation with a more accurate graphite density function is obvious.

We have verified that the barriers to migration of adsorbed rare gas atoms on graphite are very small, and it is expected that adsorbed films of rare gas atoms on graphite are nearly perfectly mobile. To have a true understanding of such films, it is necessary to investigate adatom-adatom interactions including many-body effects. We expect adatom-adatom interactions to depend most strongly on the local environment, at least in the repulsive region and near the adatom-adatom potential minimum, and the GK method should be suitable for such a study. We give the results of such a calculation separately. ${ }^{34}$

\section{ACKNOWLEDGMENTS}

I would like to thank Dr. G. S. Painter and P rofessor D. E. Ellis for providing me with the graphite wavefunctions used in this work. I would like to thank Dr. C. W. Kern, Dr. I. Shavitt, Dr. R. A. Craig, and Dr. Y. S. Kim for rnany useful discussions. In particular, I would like to thank Dr. Shavitt for advice on the numerical aspects of this work and Dr. Kim for providing the programs used to compute $\mathrm{Ar}_{2}$ potentials. I would also like to thank Dr. George Wolken for reading the manuscript and making some helpful suggestions.

*Present address: Department of Physics, The University of - Utah, Salt Lake City, Utah 84112.

${ }^{1}$ D. M. Young and A. D. Crowell, Physical Adsorption of Gases (Butterworths, Washington, 1962).

${ }^{2}$ W. A. Steele and M. Ross, J. Chem. Phys. 35, 850 (1961).

${ }^{3}$ W. A. Steele and M. Ross, J. Chem. Phys. 35, 862 (1961).

${ }^{4}$ W. A. Steele and M. Ross, J. Chem. Phys. 35, 871 (1961).

${ }^{5}$ H. E. Neustadter and R. J. Bacigalupi, Surf. Sci. 6, 246 (1967).

${ }^{6}$ F. Ricca, Nuovo Cimento Suppl. 5, 339 (1967).

${ }^{7}$ R. M. Barrer, Proc. R. Soc. Lond. A 161, 476 (1937). 
${ }^{8}$ A. D. Crowell and D. M. Young, Trans. Faraday Soc. 49 , 1080 (1953).

${ }^{9}$ E. L. Pace, J. Chem. Phys, 27, 1341 (1957).

${ }^{10}$ N. N. Avgul', A. V. Kiselev, I. A. Lygina, and D. P. Poshkus, Bull. Acad. Sci. USSR Div. Chem. Sci. (English translation) 1155 (1959).

${ }^{11}$ A. D. Crowell and R. B. Steele, J. Chem. Phys. 34, 1347 (1961).

${ }^{12}$ A. D. Crowell, J. Chem. Phys. 29, 446 (1958).

${ }^{13}$ J. R. Sams, Jr., Trans. Faraday Soc. 60, 149 (1964).

${ }^{14}$ W. A. Steele, Surf. Sci. 36, 317 (1973).

${ }^{15}$ A. J. Bennett, Phys. Rev. B 9, 741 (1974).

${ }^{16}$ B. E. F. Fender and G. D. Halsey, J. Chem. Phys. 36, 1881 (1962)

${ }^{17}$ G. H. Hudson and J. C. McCoubrey, Trans. Faraday Soc. 56, 761 (1960).

${ }^{18}$ M. Bretz, J. G. Dash, D. C. Hickernell, E. O. McLean, and O. E. Vilches, Phys. Rev. A 8, 1589 (1973).

${ }^{19}$ R. L. Elgin and D. L. Goodstein, Phys. Rev. A 9, 2657 (1974).

${ }^{20}$ C. E. Campbell, F. J. Milford, A. D. Novaco, and M. Schick, Phys. Rev. A 6, 1648 (1972).

${ }^{21}$ P. Hohenberg and W. Kohn, Phys, Rev, B 136, 864 (1964).
${ }^{22}$ N. D. Lang, in Solid State Physics, edited by H. Ehrenreich, F. Seitz, and D. Turnbull (Academic, New York, 1973), Vol. 28 , p. 225 .

${ }^{23}$ J. R. Smith, S. C. Ying, and W. Kohn, Phys. Rev. Lett. 30, 610 (1973).

${ }^{24}$ G. G. Kleiman and U. Landman, Phys. Rev. Lett. 31, 707 (1973).

${ }^{25}$ G. G. Kleiman and U. Landman, Phys. Rev. B 8, 5484 (1973).

${ }^{26}$ R. G. Gordan and Y. S. Kim, J. Chern. Phys. 56, 3122 (1972).

${ }^{27}$ Y. S. Kim, Ph. D. thesis, Harvard University, 1973.

${ }^{28}$ S. Green, J. Chem. Phys. 60, 2654 (1974).

${ }^{29}$ Y. S. Kim and R. G. Gordon, J. Chem. Phys. 60, 1842 (1974).

${ }^{30} \mathrm{~S}$. Huzinaga, D. McWilliams, and B. Domsky, J. Chem. Phys. 54, 2283 (1971).

${ }^{31}$ E. Clementi, IBM J. Res. Dev. Suppl. 9, 2 (1965).

${ }^{32}$ G. S. Painter and D. E. Ellis, Phys. Rev. B 1, 4747 (1970).

${ }^{33}$ The parameters given in Ref. 14 are slightly different than Steele actually used in his calculations. For $A=\sigma_{s s} / 2.46$, Steele actually used 1.394 rather than 1.38. We used 1.394. For $\epsilon_{p s} / k$, we used the same values as given in Ref. 14. We would like to thank Professor Steele for sending us the corrected parameters.

${ }^{34}$ D. L. Freeman (unpublished). 